PROCEEDINGS OF THE

AMERICAN MATHEMATICAL SOCIETY

Volume 126, Number 9, September 1998, Pages 2623-2630

S $0002-9939(98) 04707-8$

\title{
UNIQUENESS IN THE CAUCHY PROBLEMS FOR HIGHER ORDER ELLIPTIC DIFFERENTIAL OPERATORS
}

\author{
WENSHENG WANG
}

(Communicated by J. Marshall Ash)

\begin{abstract}
In this note, we study the uniqueness in Cauchy problems for a class of higher order elliptic differential operators with Lipschitz coefficients. In particular, we prove the uniqueness under assuming the potentials being $L_{\mathrm{loc}}^{r_{j}}$ with certain correct numbers $r_{j}$ 's.
\end{abstract}

Notation. Let $\Omega$ be a domain in $R^{d}$. Suppose $P(x, D)=\sum_{|\alpha|=m} a_{\alpha}(x) D^{\alpha}$ is a differential operator of degree $m$ with real functions $a_{\alpha}(x)$ on $\Omega$. We denote by $P=P(x, \cdot+i k)$ the symbol of $P(x, D)$ and by $N_{(x, k)}^{P}$ the zero set of $P(x, \cdot+i k)$ for any $(x, k) \in \Omega \times R^{d}$. Let's define a subset in $\Omega \times S^{d-1}$ :

$$
\begin{aligned}
\Sigma_{P}=\left\{(x, k) \in \Omega \times S^{d-1}: \sum \frac{d P}{d z_{j}}(x, \xi+i k) \cdot k_{j}\right. & \neq 0, \\
& \left.\operatorname{det} \operatorname{ess}_{C} P(x, \xi+i k) \neq 0 \forall \xi \in N_{(x, k)}^{P}\right\}
\end{aligned}
$$

where $\operatorname{Hess}_{C} P=\left(\frac{d^{2} P}{d z_{j} d z_{l}}\right)$ is the complex Hessian matrix of $P$, and $z=\xi+i k \in C^{d}$.

If $u$ is a function on $\Omega$, we define its normal support $N(\operatorname{supp} u)$ as a subset of $\Omega \times S^{d-1}$. Say $(x, k) \in N(\operatorname{supp} u)$ if there is a neighborhood $V$ of $x$ such that $\psi(y) \leq \psi(x)$ for all $y \in V \cap \operatorname{supp} u$ and $d \psi(x)= \pm k$, where $\psi$ is some smooth function.

Let $s=\frac{2(d+1)}{d+3}$ be the restriction number and $s^{\prime}$ be its conjugate number. We let $W^{m, 2}$ be the Sobolev space of functions whose derivatives up to order $m$ belong to $L^{2}$. We have the following theorem.

Theorem. Suppose $P(x, D)$ is an elliptic differential operator with real Lipschitz functions $a_{\alpha}$ as coefficients on $\Omega$ and is of order $m<\frac{d}{s}$. If a function $u \in W_{\mathrm{loc}}^{m, 2}(\Omega)$ satisfies

$$
|P u(x)| \leq \sum_{0<\mu \leq m} V_{\mu}\left|\nabla^{m-\mu} u\right|
$$

with $V_{\mu} \in L_{\text {loc }}^{\frac{d}{\mu}}(\Omega)$, then $N(\operatorname{supp} u) \subset \Sigma_{P}^{c}$.

Remarks. (1) Actually we will prove that $N(\operatorname{supp} u) \subset \Lambda_{P}^{c}$ where $\Lambda_{P}$ is the set of $(x, k) \in \Omega \times S^{d-1}$ such that $N_{(x, k)}^{P}$ is locally contained in a smooth hypersurface with nonzero Gaussian curvature, which is smaller than $\Sigma_{P}$. In other words, we

Received by the editors May 27, 1993.

1991 Mathematics Subject Classification. Primary 35Jxx.

(C)1998 American Mathematical Society 
may replace the assumptions in $\Sigma_{P}$ by directly assuming some curvature condition for $N_{x, k}^{P} . \Sigma_{P}$ is a natural condition and is easy to verify. But the proof of $\Sigma_{P} \subset \Lambda_{P}$ is nontrivial which is essentially shown in Lemma 1 below. For more details, see [3].

(2) When coefficients are constants, this theorem was proved by the author in [3]. When $P(x, D)$ is hyperbolic, under some other curvature assumption for $N_{x, k}^{P}$, Sogge proves the same result in the case where $V_{\mu}=0$ for all $\mu \leq m-1$; see [2]. In general, if we don't care about the optimal condition for the potentials, this is an old theorem by Calderon. See [1], [4].

Calderon's theorem is actually equivalent to the following uniqueness theorem in the Cauchy problem.

Theorem 1. Suppose $P(x, D)$ is an elliptic differential operator with real Lipschitz functions $a_{\alpha}$ as coefficients on a domain $\Omega$ which contains $R^{d} \backslash B\left(-e_{d}, \frac{1}{2}\right)$ and satisfies the conditions

$$
\begin{gathered}
\frac{d P}{d z_{d}}\left(0, \xi+i e_{d}\right) \neq 0, \\
\operatorname{det} \operatorname{ess}_{C} P\left(0, \xi+i e_{d}\right) \neq 0
\end{gathered}
$$

for all $\xi \in N_{\left(0, e_{d}\right)}^{P}$, where $\operatorname{Hess}_{C} P$ is the complex Hessian matrix of $P$. Then for any function $u \in W_{\mathrm{loc}}^{m, 2}(\Omega)$ satisfying (1) for some $V_{\mu} \in L_{\mathrm{loc}}^{\frac{d}{\mu}}(\Omega)$, u vanishes in a neighborhood of 0 if $u$ vanishes outside $B\left(-e_{d}, 1\right)$.

Let's first prove our Theorem by assuming Theorem 1.

Proof of the Theorem. Let $\left(x^{0}, k^{0}\right) \in N(\operatorname{supp} u)$. Suppose $\left(x^{0}, k^{0}\right) \in \Sigma_{P}$. By the definition of $N(\operatorname{supp} u)$, there is a little ball $B$ such that $x^{0} \in \partial B$ and $u=0$ in $B$. Then there is a map $F$ which is the composition of translation, rotation, dilation and Kelvin transformation with respect to $x^{0}$ and $B$ such that $F\left(x^{0}\right)=0$ and $F\left(k^{0}\right)=e_{d}$. Moreover $u \circ F^{-1}=0$ outside $B\left(-e_{d}, 1\right)$ and $u \circ F^{-1}$ is defined on a domain $\Omega$ which contains $R^{d} \backslash B\left(-e_{d}, \frac{1}{2}\right)$. Let $v(y)=u \circ F^{-1}(y)$. Then $v$ satisfies the following differential inequality by $(1)$ :

$$
|Q(y, D) v(y)| \leq \sum_{0<\mu \leq m} V_{\mu}^{1}(y)\left|\nabla^{m-\mu} v(y)\right|
$$

where $Q(y, \eta)=P\left(F^{-1}(y),\left({ }^{t} D F^{-1}(y)\right)^{-1} \eta\right)$ and $V_{\mu}^{1}(y)=V_{\mu} \circ F^{-1}(y)$ plus some bounded functions. So one may check that $\left(0, e_{d}\right) \in \Sigma_{Q}$ which means the assumptions in Theorem 1 are satisfied. So applying Theorem 1 to $Q$ and $v$, we have $v=0$ in a neighborhood of 0 . Pull back $v$ to $u$ by $F$. We have $u=0$ in a neighborhood of $x^{0}$. This is a contradiction with $x^{0} \in \operatorname{supp} u$.

In order to prove Theorem 1, we need several lemmas. Let's first study the differential operator with real constants coefficients. We denote by $A$ the vector $\left(a_{\alpha}\right)_{|\alpha|=m} \in R^{M}$ for some number $M$ determined by $m$ and $P_{A}(D)=\sum_{|\alpha|=m} a_{\alpha} D^{\alpha}$, and denote by $N_{(A, k)}$ the zero set of $P_{A}(\cdot+i k)$. We are always interested in the case that $P_{A}$ is elliptic. Let's introduce some functions as follows:

$$
S(A, \xi, k)=\left|\sum_{j} \frac{d P_{A}}{d z_{j}}(\xi+i k) k_{j}\right|,
$$




$$
\begin{gathered}
H(A, \xi, k)=\left|\operatorname{det}\left(\frac{d^{2} P_{A}}{d z_{j} d z_{l}}(\xi+i k)\right)\right| \\
L(A, \xi, k)=\sum_{(j, l)}\left|\operatorname{det}\left(\begin{array}{cc}
\frac{d \mathrm{re} P_{A}}{d \xi_{j}}(\xi+i k) & \frac{d \mathrm{re} P_{A}}{d \xi_{l}}(\xi+i k) \\
\frac{d \operatorname{im} P_{A}}{d \xi_{j}}(\xi+i k) & \frac{d \mathrm{im} P_{A}}{d \xi_{l}}(\xi+i k)
\end{array}\right)\right| .
\end{gathered}
$$

We notice that the assumption in Theorem 1 says that when $A=\left(a_{\alpha}(0)\right)$ and $k=e_{d}$, the first two of the above functions are positive on $N_{\left(0, e_{d}\right)}^{P}$. By the CauchyRiemann equation and the transversality theorem, we proved that $L(A, \xi, k)$ is also positive on $N_{\left(0, e_{d}\right)}^{P}$. See [3].

Lemma 1. Suppose for some $A \in R^{M}$ and $k_{0} \in S^{d-1}$ the above three functions are positive on $N_{\left(A, k_{0}\right)}$. Then there are some positive numbers $c_{0}, b, \epsilon$, an integer $J, a$ neighborhood $K$ of $k_{0}$ in $S^{d-1}$ and finite small balls $\left\{B_{j}(\epsilon)\right\}_{j=1}^{J}$ such that for any $B \in R^{M}$ with $\|B-A\| \leq b$ and any $k \in K$ there are finite hypersurfaces $\left\{S_{j}\right\}_{j=1}^{J}$ for which the following properties hold:

(1) $N_{(B, k)} \cap B_{j}(\epsilon) \subset S_{j} \cap B_{j}(\epsilon)$;

(2) $N_{(B, k)} \subset \bigcup_{j=1}^{J} B_{j}\left(\frac{\epsilon}{2}\right)$;

(3) $S_{j} \cap B_{j}(\epsilon)$ is a piece of hypersurface with nonzero Gaussian curvature which is bounded by $c_{0}$ from below for all $j$.

Moreover for each such $(B, k)$, there is a diffeomorphism $G_{(B, k)}: \bigcup_{j=1}^{J} B_{j}(\epsilon) \rightarrow$ $D(\epsilon) \times N_{(B, k)}$ such that $\left|G_{(B, k)}^{\prime}\right|$ is bounded by $c_{0}$ from below.

Proof. We will prove this lemma in several steps as follows.

Step 1: There are positive constants $c, b$ and a neighborhood $K$ of $k_{0}$ in $S^{d-1}$ and an $\epsilon$ neighborhood $U$ of $N_{\left(A, k_{0}\right)}$ such that for any $B \in R^{M}$ with $\|B-A\| \leq b$ and any $k \in K$,

for all $\xi \in U$.

$$
\begin{gathered}
N_{(B, k)} \subset \frac{1}{2} U, \\
\min (S(B, \xi, k), H(B, \xi, k), L(B, \xi, k)) \geq c
\end{gathered}
$$

Proof of Step 1. Since $P_{A}$ is an elliptic polynomial, the set $N_{\left(A, k_{0}\right)}$ is a compact boundaryless submanifold of codim 2 by assumption. Functions $S, H$ and $L$ are continuous in three variables $A, \xi$ and $k$. So by assumption and compact argument and the $\epsilon$ neighborhood theorem, Step 1 is proved.

Step 2: There are $\epsilon$ and finite small balls such that for any $B$ and $k$ as in Step 1 there are finite hypersurfaces as in Lemma 1. (1), (2) and (3) of Lemma 1 hold.

Proof of Step 2 . Since $S\left(A, \xi, k_{0}\right)$ and $H\left(A, \xi, k_{0}\right)$ are positive functions, Proposition 0.1 of [3] implies that there are finite $\epsilon$ balls $\left\{B_{j}(\epsilon)\right\}_{j=1}^{J}$ with centers $\left\{\xi_{j}\right\} \subset$ $N_{\left(A, k_{0}\right)}$ such that

$$
N_{\left(A, k_{0}\right)} \subset \bigcup_{j=1}^{J} B_{j}\left(\frac{\epsilon}{4}\right) .
$$

Moreover there are also finite real numbers $t_{j}$ and vectors $\left\{x_{j}\right\}_{j=1}^{J} \subset R^{d}$ such that if we define functions $f_{j}\left(A, \xi, k_{0}\right)$ by

$$
\begin{gathered}
\operatorname{re} P_{A}\left(\xi+i k_{0}\right)+t_{j} \operatorname{im} P_{A}(\xi+i k)+\left\langle x_{j}, \xi-\xi_{j}\right\rangle\left(t_{j} \operatorname{re} P_{A}\left(\xi+i k_{0}\right)-\operatorname{im} P_{A}(\xi+i k)\right) \\
-\left\langle x_{j}, \xi-\xi_{j}\right\rangle\left(\operatorname{re} P_{A}\left(\xi+i k_{0}\right)+t_{j} \operatorname{im} P_{A}(\xi+i k)\right) \\
\times \frac{\left\langle t_{j} \nabla \operatorname{re} P_{A}\left(\xi_{j}+i k_{0}\right)-\nabla \operatorname{im} P_{A}\left(\xi_{j}+i k_{0}\right), \nabla \operatorname{re} P_{A}\left(\xi_{j}+i k_{0}\right)+t_{j} \nabla \operatorname{im} P_{A}\left(\xi_{j}+i k_{0}\right)\right\rangle}{\left\langle\nabla \operatorname{re} P_{A}\left(\xi_{j}+i k_{0}\right)+t_{j} \nabla \operatorname{im} P_{A}\left(\xi_{j}+i k_{0}\right), \nabla \operatorname{re} P_{A}\left(\xi_{j}+i k_{0}\right)+t_{j} \nabla \operatorname{im} P_{A}\left(\xi_{j}+i k_{0}\right)\right\rangle},
\end{gathered}
$$


then $f_{j}\left(A, \cdot, k_{0}\right)^{-1}(0)$ is a hypersurface with Gaussian curvature bounded by $2 c_{0}$ from below in $B_{j}(\epsilon)$ for some constant $c_{0}$ which depends only on $A$ and $k_{0}$. Now let's fix a $B$ and a $k$ as in Step 1 . When $b$ and $K$ are small enough, $N_{(B, k)} \subset \bigcup_{j=1}^{J} B_{j}\left(\frac{\epsilon}{2}\right)$. Choose $\eta_{j} \in B_{j}\left(\frac{\epsilon}{2}\right)$ with $P_{B}\left(\eta_{j}+i k\right)=0$. Replace $A, k_{0}$ and $\xi_{j}$ by $B, k$ and $\eta_{j}$ in the function $f_{j}$ for each $j$. Then once again when $b$ and $K$ are small enough, $f_{j}(B, \cdot, k)^{-1}(0) \cap B_{j}(\epsilon)$ is a piece of hypersurface with Gaussian curvature bounded by $c_{0}$ from below for all $j$. This proves Step 2 with $S_{j}=f_{j}(B, \cdot, k)^{-1}(0)$.

Step 3: The last part in Lemma 1 holds when $\epsilon$ is small and $J$ is larger.

Proof of Step 3. By the $\epsilon$ neighborhood theorem, when $\epsilon$ is small and $J$ is large, there is a diffeomorphism $G_{\left(A, k_{0}\right)}: \bigcup B_{j}(2 \epsilon) \rightarrow D(2 \epsilon) \times N_{\left(A, k_{0}\right)}$ where $D(2 \epsilon)$ is a 2-dimensional ball of radius $2 \epsilon$. In fact $G_{\left(A, k_{0}\right)}$ may be defined by extending $N_{(B, k)}$ along the normal directions, which we may choose as $\nabla \operatorname{re} P_{A}\left(\xi+i k_{0}\right)+$ $t_{j} \nabla \operatorname{im} P_{A}\left(\xi+i k_{0}\right)$ and $t_{j} \nabla \operatorname{re} P_{A}\left(\xi+i k_{0}\right)-\nabla \operatorname{im} P_{A}\left(\xi+i k_{0}\right)-v$ where $v$ is the projection of $t_{j} \nabla \operatorname{re} P_{A}\left(\xi+i k_{0}\right)-\nabla \operatorname{im} P_{A}\left(\xi+i k_{0}\right)$ in the $\nabla \operatorname{re} P_{A}\left(\xi+i k_{0}\right)+$ $t_{j} \nabla \operatorname{im} P_{A}\left(\xi+i k_{0}\right)$ direction in each $B_{j}\left(\frac{1}{2} \epsilon\right)$. Since $P_{B}(\xi+i k)$ are smooth in $(B, \xi, k)$ and $L(B, \xi, k) \geq c$ by the assumption, for each $B$ closing $A$ and each $k$ closing $k_{0}$, there is a diffeomorphism $G_{(B, k)}: \bigcup B_{j}(\epsilon) \rightarrow D(\epsilon) \times N_{(B, k)}$ such that $\left|G_{(B, k)}^{\prime}\right|$ is bounded by $\frac{1}{2}\left|G_{\left(A, k_{0}\right)}^{\prime}\right|$ from below. This proves Step 3.

Finally if we let $c_{0}$ be a new constant decided by Step 2 and Step 3, we prove Lemma 1.

Let $\Gamma$ be the open cone such that $\Gamma \cap S^{d-1}=K$ which is as in Lemma 1. If $E$ is a compact convex set with interior, then we define

$$
g_{E}(x)=\min (T \geq 1: x \in T E) .
$$

Fix once and for all $t>d$, and define $\|u\|_{p, E}=\left\|g_{E}^{t} u\right\|_{p}$. Then by the Holder inequality we have

$$
\|u\|_{p} \leq C|| u \|_{q, E}|E|^{\frac{1}{p}-\frac{1}{q}}
$$

for any $q \geq p$, where $C$ depends only on $t$ and $d$.

Lemma 2. Suppose $P_{A}$ is as in Lemma 1 and is of order $m<\frac{d}{s}$. Let $b$ and $\Gamma$ be as before or as in Lemma 1. Then there is a constant $C_{A}$ such that for all $B \in R^{M}$ with $\|B-A\| \leq b$ and any $k \in \Gamma$ and all compact convex subsets $E \subset R^{d}$ with $|E| \geq|k|^{-d}$, we have

$$
\left\|e^{k \cdot x} \nabla^{m-\mu} f\right\|_{q_{\mu}} \leq\left. C_{A}\left(|k|^{d} \mid E\right)\right|^{\frac{\mu}{d}}\left\|e^{k \cdot x} P_{B}(D) f\right\|_{2, E}
$$

for all $f \in W^{m, 2}$ with compact support and all integers $0<\mu \leq m$, where $q_{\mu}$ are the real numbers satisfying $\frac{1}{2}-\frac{1}{q_{\mu}}=\frac{\mu}{d}$. When $\mu=0$, we have the following inequality:

$$
\left\|e^{k \cdot x} \nabla^{m} f\right\|_{2} \leq C_{A}(|k| \operatorname{diam} E)\left\|e^{k \cdot x} P_{B}(D) f\right\|_{2, E} .
$$

Proof. Let $a=\left(\frac{1}{s}, 0\right), b=(1,0), c=\left(1, \frac{1}{2}\right)$ and $d=\left(\frac{1}{s}, \frac{1}{s^{\prime}}\right)$. Let $Q$ be a subset of $R^{2}$ consisting of the quadrilateral $a b c d$ and two sides $a d$ and $b c$. Let $B$ and $k$ with $|k|=1$ be as in Lemma 2. So the conclusions of Lemma 1 hold for this $(B, k)$. First let $0<\mu \leq m$.

The inequality (3) is equivalent to

$$
\left\|(m \hat{v})^{\vee}\right\|_{q_{\mu}} \leq C_{A}\left(|k|^{d}|E|\right)^{\frac{\mu}{d}}\|v\|_{2, E}
$$

with $m(\xi)=\frac{|\xi+i k|^{m-\mu}}{P_{B}(\xi+i k)}$ for all $v \in C_{0}^{\infty}$. 
Let $U_{\frac{1}{2}}=\bigcup_{j=1}^{J} B_{j}\left(\frac{\epsilon}{2}\right)$ and $U_{1}=\bigcup_{j=1}^{J} B_{j}(\epsilon)$ which are in Lemma 1 . Let $\phi$ be a smooth cutoff function taking 1 on $U_{\frac{1}{2}}$, and 0 on $U_{1}^{c}$. Write $m=m_{1}+m_{2}$ with $m_{1}=m \phi$ and $m_{2}=m(1-\phi)$. By Lemma 1, the exact proof of Lemma 2.1 in [3] shows that

$$
\left\|\left(m_{1} \hat{v}\right)^{\vee}\right\|_{q} \leq C_{A}\|v\|_{p}
$$

for all $\left(\frac{1}{p}, \frac{1}{q}\right) \in Q$, where $C_{A}$ is some constant which depends only on $A, k_{0}$ and $d$. Since $m_{2}(\xi) \leq(1+|\xi|)^{-\mu}$, by the Bessel potential theory, we have

$$
\left\|\left(m_{2} \hat{v}\right)^{\vee}\right\|_{q} \leq C_{A}\|v\|_{p}
$$

for all $\frac{1}{p}-\frac{1}{q}=\frac{\mu}{d}$. Let $q_{\mu}$ be such that $\frac{1}{2}-\frac{1}{q_{\mu}}=\frac{\mu}{d}$, and let $q_{\mu}^{1}$ be such that $\frac{1}{s}-\frac{1}{q_{\mu}^{1}}=\frac{\mu}{d}$ if $\mu \geq 2, q_{1}^{1}$ is sufficiently close to $s^{\prime}$ and is bigger than $s^{\prime}$. Then for any compact convex set $|E| \geq 1$, since $q_{\mu}^{1}<q_{\mu}$ and $m_{1}$ has compact support, we have by using (6) and (2)

$$
\left\|\left(m_{1} \hat{v}\right)^{\vee}\right\|_{q_{\mu}} \leq\left\|\left(m_{1} \hat{v}\right)^{\vee}\right\|_{q_{\mu}^{1}} \leq C_{A}\|v\|_{s} \leq C_{A}|E|^{\frac{1}{s}-\frac{1}{2}}\|v\|_{2, E}
$$

which is bounded by $C_{A}|E|^{\frac{\mu}{d}}|| v \|_{2, E}$ since $|E| \geq 1$. Combining (8) and (7) we prove (5) and hence (3) with $|k|=1$. After a scaling we prove Lemma 2 with $\mu \geq 1$.

Finally when $\mu=0$, the inequality (4) was already showed in [4] without using any curvature property in Lemma 1 . So this proves Lemma 2.

Lemma 3. Suppose $f$ is supported in a ball B. Let $D(a, N)$ be a fixed ball in $R^{d}$. Then there is a pairwise disjoint compact convex subset $\left\{E_{k_{j}}\right\}$ with $\left\{k_{j}\right\} \subset D(a, N)$ such that

$$
\begin{gathered}
\left\|e^{k_{j} \cdot x} f \cdot g_{E_{k_{j}}}\right\|_{1, E_{k_{j}}} \leq C_{0}^{2}\left\|e^{k_{j} \cdot x} f\right\|_{L^{1}\left(E_{k_{j}}\right)}, \\
\sum\left|E_{k_{j}}\right|^{-1} \geq C^{-1} N^{d}, \forall s \geq 1, \\
\operatorname{diam} E_{k_{j}} \leq C_{0} N^{-\frac{1}{2}}, \\
E_{k_{j}} \text { contains a ball of radius }\left(C_{0} N\right)^{-1}, \\
E_{k_{j}} \subset 2 B
\end{gathered}
$$

where $C_{0}$ is a universal constant depending only on $d$.

Proof. This is a special case of Wolff's measure lemma in [4].

Now let's start to prove Theorem 1. First we claim that we may assume the Lipschitz norm of $a_{\alpha}(x)$ is less than a small number $\rho$ which will be chosen later. In fact let $F^{1}(x)=\delta^{-1} x, F_{2}(x)=\left(\bar{x},-x_{d}\right), F_{3}(x)=\frac{x+e_{d}}{\left|x+e_{d}\right|^{2}}-e_{d}$ and let $F=$ $F_{3} \circ F_{2} \circ F_{1}$. Then if $\delta$ is small enough, the function $v=u \circ F^{-1}$ is defined on a domain which contains $R^{d} \backslash B\left(-e_{d}, \frac{1}{2}\right)$ and $v=0$ outside $B\left(-e_{d}, 1\right)$. Moreover $v$ satisfies the following differential inequality:

$$
\left|P_{\delta}(y, D) v(y)\right| \leq \sum_{0<\mu \leq m} V_{\mu}(y)\left|\nabla^{m-\mu} v(y)\right|
$$

where $V_{\mu}(y)$ has the same properties as before, $P_{\delta}(y, D)=\sum_{|\alpha|=m} a_{\alpha}^{\delta}(y) D^{\alpha}$ with $a_{\alpha}^{\delta}(0)=a_{\alpha}(0)$ and $\left\|a_{\alpha}\right\|_{\text {Lip }} \leq \delta\left\|a_{\alpha}\right\|_{\text {Lip }}$. Let $\rho$ be this number. On the other hand, if we let $A=\left(a_{\alpha}^{\delta}(0)\right)=\left(a_{\alpha}(0)\right)$ and $b, \Gamma$ be as in Lemma 2 or Lemma 1 with 
$k_{0}=e_{d}$, then when $\delta$ is small enough for any $y \in B\left(0, \frac{1}{2}\right)$ with $B=\left(a_{\alpha}^{\delta}(y)\right)$ the inequalities (3) and (4) hold for all small $\delta$.

Let's assume $0 \in \operatorname{supp} v$. Let $S$ be the convex hull of $\operatorname{supp} v \cap\left\{y \in R^{d}: y_{d} \geq-\frac{1}{16}\right\}$ and $\phi$ be a smooth cutoff function such that $\phi=0$ when $y_{d} \leq-\frac{1}{8}, \chi=1$ in a neighborhood of $\partial S$ and $\sum_{0<\mu m} \|\left. V_{\mu}\right|_{L^{\frac{d}{\mu}(\operatorname{supp} \phi)}} ^{\frac{d}{\mu}} \leq \beta$ with a small constant $\beta$ to be chosen later. Let $w=v \phi$. Then by (4)

$$
\left|P_{\delta}(y, D) w(y)\right| \leq \sum_{0<\mu \leq m} V_{\mu}(y)\left|\nabla^{m-\mu} w(y)\right|+\chi
$$

where $\chi \in L^{2}$ and supp $\chi \subset A^{1} \cup A_{2}$; here $A_{2}=\left\{y \in B\left(-e_{d}, 1\right):-\frac{1}{16} \geq y_{d} \geq-\frac{1}{8}\right\}$ and $A_{1}$ is a compact subset of $S$. Let $r \leq \frac{1}{32}$ be a fixed small number so that the cone $\Gamma_{r}=\left\{k \in R^{d}: k_{d}>r^{-1} \sqrt{|k|^{2}-k_{d}^{2}}\right\}$ is contained in $\Gamma$ which is as in Lemma 2 for $P_{A}$. So $r$ is independent of $\rho$.

Lemma 4. If $\tau>0$, then there is an $L_{0}$ such that if $k \in \Gamma_{r}$ and $|k| \geq L_{0}$, then

$$
\left\|e^{k \cdot y} \chi \cdot g_{E}\right\|_{2, E} \leq\left\|e^{k \cdot y} \sum_{0<\mu \leq m} V_{\mu} \mid \nabla^{m-\mu} w\right\|_{2}
$$

for all $E \subset B\left(0, \frac{1}{2}\right)$ with $E$ containing a ball of radius $\tau|k|^{-1}$.

Proof. Since $\Gamma_{r}$ has conjugate cone $\left\{k \in R^{d}:\left\langle k, k^{\prime}\right\rangle \leq 0 \forall k^{\prime} \in \Gamma_{r}\right\}$ which contains $B\left(-e_{d}, 1\right) \cap\left\{y: y_{d} \leq \frac{1}{6}\right\} \supset A_{2}$, the rest of the proof is exactly the same as the proof of Lemma 7.1 of [4]. So we are done.

Proof of Theorem 1. Let $L \geq L_{0}$ be a large number. We will apply Lemma 3 to the function

$$
f=\left(\sum_{0<\mu \leq m} V_{\mu}\left|\nabla^{m-\mu} w\right|+\rho L^{-\frac{1}{2}}\left|\nabla^{m} w\right|\right)^{2}
$$

and the ball $B\left(L e_{d}, \frac{1}{2} r L\right)$ with $a=L e_{d}$ and $N=\frac{1}{2} r L$. So $\frac{1}{2} L \leq\left|k_{j}\right| \leq 2 L$. Let $Y_{j}=E_{k_{j}} \cap \operatorname{supp} w$, let $y_{j}$ be the center of the convex set $E_{k_{j}}$ and let $B_{j}=\left(a_{\alpha}^{\delta}\left(y_{j}\right)\right)$. So we have $\left\|B_{j}-A\right\| \leq b$ and the inequalities (3) and (2) in Lemma 2. Then by using Holder's inequality, (3), (4), and (11)

$$
\begin{gathered}
\left\|e^{k_{j} \cdot y}\left(\sum_{0<\mu \leq m} V_{\mu}\left|\nabla^{m-\mu} w\right|+\rho L^{-\frac{1}{2}}\left|\nabla^{m} w\right|\right)\right\| L_{L^{2}\left(E_{k_{j}}\right)} \\
\leq \sum_{0<\mu \leq m}\left\|V_{\mu}\right\|_{L^{\frac{d}{\mu}}\left(Y_{j}\right)}\left\|e^{k_{j} \cdot y} w\right\|_{q_{\mu}}+\rho L^{-\frac{1}{2}}\left\|e^{k_{j} \cdot y} \nabla^{m} w\right\|_{2} \\
\leq C_{A}\left(\sum_{0<\mu \leq m}\left(\left|k_{j}\right|^{d}\left|E_{k_{j}}\right|\right)^{\frac{\mu}{d}}\left\|V_{\mu}\right\|_{L^{\frac{d}{\mu}}\left(Y_{j}\right)}+\rho L^{-\frac{1}{2}}\left|k_{j}\right| \operatorname{diam} E_{k_{j}}\right)\left\|e^{k_{j} \cdot y} P_{B_{j}}(D) w\right\|_{2, E_{k_{j}}}
\end{gathered}
$$

$$
\leq 2 C_{A}\left(\sum_{0<\mu \leq m}\left(L^{d}\left|E_{k_{j}}\right|\right)^{\frac{\mu}{d}}\left\|V_{\mu}\right\|_{L^{\frac{d}{\mu}}\left(Y_{j}\right)}+C_{0} r^{-1} \rho\right)\left\|e^{k_{j} \cdot y} P_{B_{j}}(D) w\right\|_{2, E_{k_{j}}} .
$$


On the other hand, since $a_{\alpha}^{\delta}$ is Lipschitz continuous it follows that $\left|a_{\alpha}^{\delta}\left(y_{j}\right)-a_{\alpha}^{\delta}(y)\right| \leq$ $\rho \cdot\left|y_{j}-y\right| \leq \rho \operatorname{diam} E_{k_{j}} g_{E_{k_{j}}} \leq C_{0} r_{-1} \rho L^{-\frac{1}{2}} g_{E_{k_{j}}}$ by (11). So

$$
\left|P_{B_{j}}(D) w(y)\right| \leq\left|P_{\delta}(y, D) w(y)\right|+C_{0} r^{-1} \rho L^{-\frac{1}{2}} g_{E_{k_{j}}}\left|\nabla^{m} w\right|
$$

and hence by (13)

$$
\left|P_{B_{j}}(D) w(y)\right| \leq \sum_{0<\mu \leq m} V_{\mu}\left|\nabla^{m-\mu} w\right|+C_{0} r^{-1} \rho L^{-\frac{1}{2}} g_{E_{k_{j}}}\left|\nabla^{m} w\right|+\chi .
$$

Because of (14), we may ignore the term $\chi$ in the following process. Now by using (9) we have

$$
\begin{aligned}
& \left\|e^{k_{j} \cdot y} P_{B_{j}}(D) w\right\|_{2, E_{k_{j}}} \\
& \quad \leq 2 C_{0} r^{-1}|| e^{k_{j} \cdot y}\left(\sum_{0<\mu \leq m} V_{\mu}\left|\nabla^{m-\mu} w\right|+\rho L^{-\frac{1}{2}}\left|\nabla^{m} w\right|\right) g_{E_{k_{j}}} \|_{2, E_{k_{j}}} \\
& \quad \leq 2 C_{0}^{2} r^{-1}|| e^{k_{j} \cdot y}\left(\sum_{0<\mu \leq m} V_{\mu}\left|\nabla^{m-\mu} w\right|+\rho L^{-\frac{1}{2}}\left|\nabla^{m} w\right|\right) \|_{L^{2}\left(E_{k_{j}}\right)} .
\end{aligned}
$$

So combining (15) and (16), we have

$$
1 \leq 2 C_{0}^{2} r^{-1} \cdot 2 C_{A}\left(\sum_{0<\mu \leq m}\left(L^{d}\left|E_{k_{j}}\right|\right)^{\frac{\mu}{d}}\left\|V_{\mu}\right\|_{L^{\frac{d}{\mu}}\left(Y_{j}\right)}+C_{0} r^{-1} \rho\right) .
$$

Remember the constants $r, C_{0}$ and $C_{A}$ are independent of $\rho$, i.e., $\delta$. So after making $\delta$ and hence $\rho$ small, (17) implies

$$
\sum_{0<\mu \leq m}\left(L^{d}\left|E_{k_{j}}\right|\right)^{\frac{\mu}{d}}\left\|V_{\mu}\right\|_{L^{\frac{d}{\mu}}\left(Y_{j}\right)} \geq C
$$

and hence

$$
\max _{0<\mu \leq m}\left\{\|\left. V_{\mu}\right|_{L^{\frac{d}{\mu}}\left(Y_{j}\right)} ^{\frac{d}{\mu}}\right\} \geq C\left(L^{d}\left|E_{k_{j}}\right|\right)^{-1}
$$

for some constant $C$ depending only on $d$ and $A$. Summing up over $j$ for (18), (10) implies that

$$
\sum_{0<\mu \leq m}\left\|V_{\mu}\right\|_{L^{\frac{d}{\mu}}(\operatorname{supp} w)}^{\frac{d}{\mu}} \geq C_{0}^{-1} C
$$

which is a contradiction if $\beta$ is small enough. This proves Theorem 1.

\section{ACKNOWLEDGMENTS}

I would like to thank Professor Tom Wolff for his constant encouragement while I was considering this problem. I would also like to thank the Alfred P. Sloan Foundation for a Doctoral Dissertation Fellowship, 1992-93. 


\section{REFERENCES}

1. A. P. Calderon, Uniqueness in the Cauchy problem for partial differential equations, Amer. J. Math., 80 (1958) 16-36. MR 21:3675

2. C. Sogge, Uniqueness in Cauchy problem for hyperbolic differential operator, Trans. of AMS., 333 (1992) 821-833. MR 92m:35006

3. W. Wang, Carleman inequalities and unique continuation for higher order elliptic differential operators, Duke Math. J., 74 (1994) 107-128. MR 95j:35078

4. T. Wolff, A property of measures in $R^{n}$ and an application to unique continuation, Geometrical and Functional Analysis, 2 (1992) 225-284. MR 93c:35015

Department of Mathematics, Florida International University, Miami, Florida 33199

E-mail address: wangwens@zeus.fiu.edu or wangw@solix.fiu.edu 